\title{
Simultaneous Measurement of Distance and Thickness of a Thin Metal Plate With an Electromagnetic Sensor Using a Simplified Model
}

\author{
Wuliang Yin, A. J. Peyton, and Stephen J. Dickinson
}

\begin{abstract}
This paper presents a simplified model which can describe the inductance change when an air-core coil is placed next to a thin nonmagnetic metallic plate. The model has two independent parameters and is valid for a range of thickness, conductivity, and lift-offs. Use of this new relationship provides a fast and accurate method to measure the distance and thickness simultaneously. Measurements made for a sample coil next to thin copper and aluminum plates of various thicknesses verified the theory and the proposed method.
\end{abstract}

Index Terms-Distance and thickness measurement, electromagnetic sensors, frequency-dependent response, simplified model.

\section{INTRODUCTION}

Q UALITY inspection and thickness measurement of metal plates using eddy current methods are affected by lift-off variations. Furthermore, simultaneous thickness and distance measurements of metal layers are required for a range of technological applications such as coating and surface treatment [1]-[5]. A variety of coil designs have been used in these applications. In method [2], a ferrite coil sensor was developed to measure the thickness and distance of a zinc-aluminum coating on a steel sheet. A double air-cored coil method was proposed to eliminate the undesirable lift-off effect [3].

In most of these methods, theoretical impedance values were computed for a variety of possible combination of the parameter values, a set of parameters for which the theoretical data was as close as possible to the experimental data was chosen. This usually involves a simplex search method. For a large number of parameter values, the search process is time consuming. A feature-based inversion method for conductivity and thickness measurement greatly reduced the time [4]. However, these features are still coupled together and therefore a multidimensional look-up table has to be constructed to inverse the parameters.

In this paper, we have found that for an air-cored coil next to a nonmagnetic thin metallic plate, two features of imaginary part of the inductance values link to the thickness and distance of the plate, respectively. Based on this finding, a rapid method of simultaneous measuring the thickness and distance is proposed.

Manuscript received June 15, 2003; revised April 5, 2004. This work was supported by the U.K. Engineering and Physical Sciences Research Council (EPSRC).

The authors are with the Engineering Department, Lancaster University, Lancaster, LA1 4YR, U.K. (e-mail: w.yin@lancater.ac.uk).

Digital Object Identifier 10.1109/TIM.2004.830585
For an air-core coil, the general analytical solution for inductance change caused by a thin layer of nonmagnetic metal was described by Dodd and Deeds [6]. This paper evaluated the analytical solutions of Dodd and Deeds for nonmagnetic plates of different thickness, conductivity, and lift-offs. It has been found that the solution can be approximated by a simple scaling relation.

$$
\Delta L(\omega)=\Delta L_{0} \cdot L^{*}\left(\frac{\omega}{\omega_{0}}\right) .
$$

Here, $\Delta L_{0}$ is the overall strength of the inductance change that depends on lift-off for a given coil, and is independent of the thickness and conductivity, $\omega_{0}$ is the characteristic angular frequency at which the imaginary part of $\Delta L_{0}$ is a maximum. The frequency $\omega_{0}$ depends on the conductivity and thickness of the plate and is virtually independent of lift-off. The function $L^{*}\left(\omega / \omega_{0}\right)$ is

$$
L^{*}\left(\frac{\omega}{\omega_{0}}\right)=\frac{\left(j \frac{\omega}{\omega_{0}}\right)}{\left(1+j \frac{\omega}{\omega_{0}}+0.15 j \sqrt{\frac{\omega}{\omega_{0}}}\right)} .
$$

Measurements were made for a sample coil next to thin copper and aluminum plates of various thicknesses using an impedance analyzer (SL1260, Solartron). These results can be approximated by (1) and (2) to reasonable precision. Comparison between the calculated and measured peak $\operatorname{Im} \Delta L_{0}$ and $\omega_{0}$ provides the lift-off and thickness simultaneously.

\section{MethodS}

This section describes the procedures used to determine the simplified model. First, the two limiting cases for $\Delta L(\omega)$ with $\omega=0$ and $\infty$ will be considered with a physical explanation of the results. Then, the calculated and measured results will be displayed. Further, the procedure to simplify the analytic solution of Dodd and Deeds will be given. Finally, simultaneous thickness and lift-off inferred from measurements are listed.

\section{A. Analytic Solution}

The difference in the complex inductance is $\Delta L(\omega)=$ $L(\omega)-L_{A}(\omega)$, where $L(\omega)$ is the coil inductance above a plate, and $L_{A}(\omega)$ is the inductance in free space. Fig. 1 is a schematic diagram of the model.

The formulas of Dodd and Deeds [6] are

$$
\Delta L(\omega)=K \int_{0}^{\infty} \frac{P^{2}(\alpha)}{\alpha^{6}} A(\alpha) \phi(\alpha) d \alpha
$$




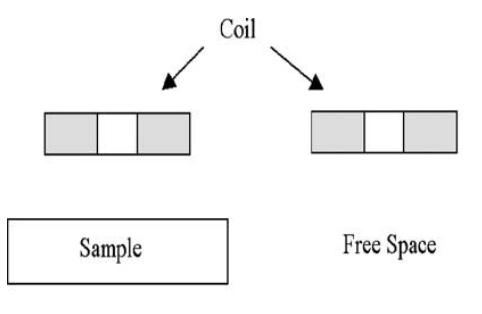

(a)

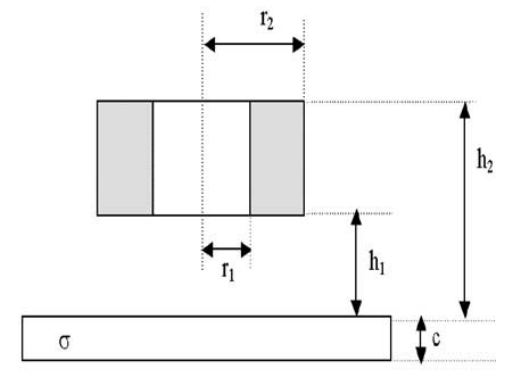

(b)

Fig. 1. (a) Schematic diagram of the model. (b) Geometry of the coil.
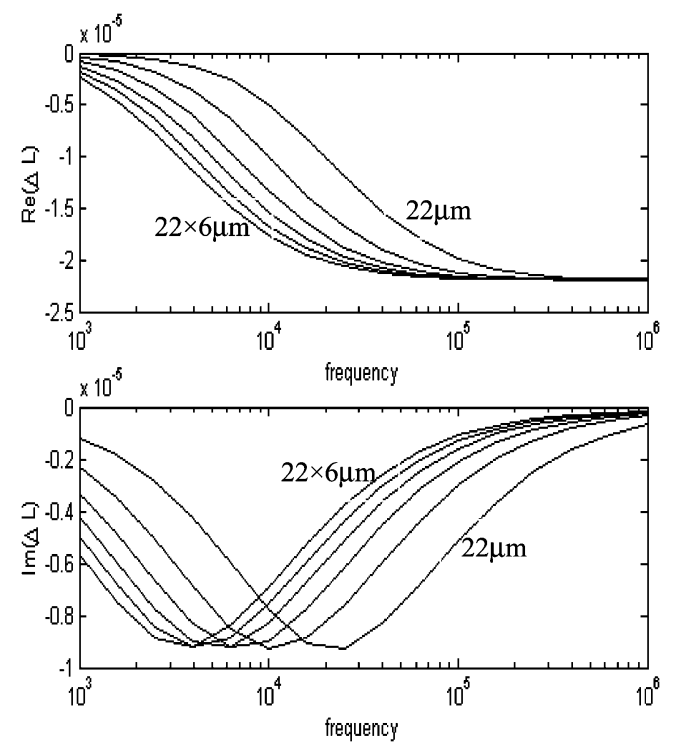

Fig. 2. The real and imaginary parts of $\Delta L_{0}$ for copper plates with thickness $22 \mu \mathrm{m}, 44 \mu \mathrm{m}, \ldots 22 \times 6 \mu \mathrm{m}$.

where

$$
\begin{aligned}
\phi(\alpha) & =\frac{\left(\alpha_{1}+\alpha\right)\left(\alpha_{1}-\alpha\right)-\left(\alpha_{1}+\alpha\right)\left(\alpha_{1}-\alpha\right) e^{2 \alpha_{1} c}}{-\left(\alpha_{1}-\alpha\right)\left(\alpha_{1}-\alpha\right)+\left(\alpha_{1}+\alpha\right)\left(\alpha_{1}+\alpha\right) e^{2 \alpha_{1} c}} \\
\alpha_{1} & =\sqrt{\alpha^{2}+j \omega \sigma \mu_{0}} \\
K & =\frac{\pi \mu_{0} N^{2}}{\left(l_{1}-l_{2}\right)^{2}\left(r_{1}-r_{2}\right)^{2}} \\
P(\alpha) & =\int_{\alpha r_{1}}^{\alpha r_{2}} x J_{1}(x) d x, \quad A(\alpha)=\left(e^{-\alpha l_{1}}-e^{-\alpha l_{2}}\right)^{2}
\end{aligned}
$$

where $\mu_{0}$ denotes the permeability of free space. $N$ denotes the number of turns in the coil; $r_{1}$ and $r_{2}$ denote the inner and outer radii of the coil, while $l_{1}$ and $l_{2}$ denote the height of the bottom and top of the coil; and $c$ denotes the thickness of the plate.
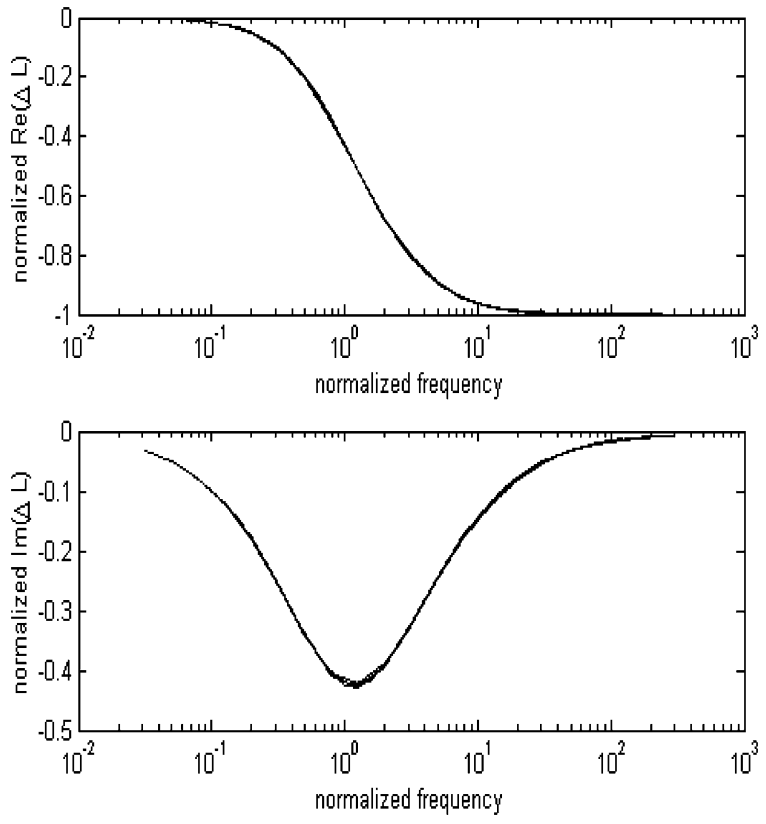

Fig. 3. The six curves in Fig. 2 overlap identically when plotted verse normalized frequency.

\section{B. $\Delta L(\omega)$ in Two Limiting Cases $\omega=0$ and $\infty$}

Setting $\omega=0$ gives the inductance change for zero-frequency. The real part and imaginary part of the inductance change are zero, which means the nonmagnetic plate causes no inductance change and the magnetic flux penetrates the plate as in free space.

In the limit of arbitrary large frequency, the inductance change is given by $\Delta L=-\Delta L_{0}$, where $\Delta L_{0}=$ $K \int\left(P^{2}(\alpha) / \alpha^{6}\right) A(\alpha) d \alpha . \Delta L_{0}$ is dependent on lift-off for a given coil, and is independent of the thickness and conductivity, which corresponds to the situation that the incident magnetic flux is totally excluded from the plate.

\section{The Scaling Relation With Various Calculated Results and Measured Results}

The frequency-dependent inductance change for a coil defined by $r_{1}=20 \mathrm{~mm}, r_{2}=20.1 \mathrm{~mm}, h=2 \mathrm{~mm}$, and $N=10$ is plotted in Fig. 2 . The conductivity of the plate was chosen to be $5.8 \times 10^{7}$ (copper) and thickness was chosen to be $22 \mu \mathrm{m}, 22 \times 2 \mu \mathrm{m}, \ldots 22 \times 6 \mu \mathrm{m}$. The lift-off was chosen to be $1 \mathrm{~mm}$.

On examination of Fig. $2, \operatorname{Im} \Delta L_{0}$ peaks at a characteristic frequency $\omega_{0}$, which is seen to increase as thickness decreases. Fig. 3 shows the same calculations scaled by $1 / \Delta L_{0}$ versus the normalized frequency. All the calculations fall on the same curve. On trials over a range of conductivities and thicknesses, it was found that this common scaling relation could also be applied to calculations for plates with different conductivity (e.g., $3.82 \times 10^{7} \Omega$ for aluminum) and different lift-offs $(1 \mathrm{~mm}$, $1.1 \mathrm{~mm} . .2 .0 \mathrm{~mm}$ ), the existence of this common scaling relation was also verified by measurements from a coil (parameters defined earlier) with aluminum and copper foil samples (see Fig. 4). 

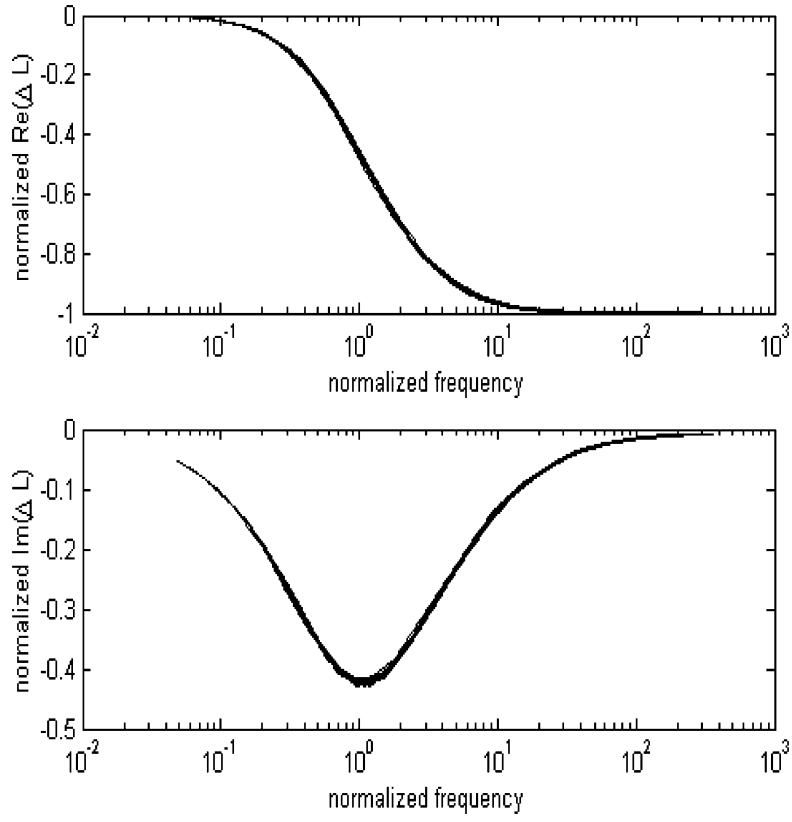

Fig. 4. Measurements for aluminum and copper foil samples overlap identically when plotted verse normalized frequency.

\section{The Simplification of the Analytic Solution}

Two approximations can be used to simplify the analytical solution.

Substituting $e^{2 \alpha_{1} c}$ with $1+2 \alpha_{1} c$, and considering (5) and (4) becomes

$$
\phi(\alpha) \approx \frac{j \omega \sigma \mu_{0} c}{j \omega \sigma \mu_{0} c+2 \alpha^{2} c+2 \alpha+2 \alpha \alpha_{1} c} .
$$

The next approximation originates from the fact that $\phi(\alpha)$ varies slowly with $\alpha$ compared to the rest of the integrand, which reaches its maximum at a characteristic spatial frequency $\alpha_{0} . \alpha_{0}$ is defined to be one over the smallest dimension of the coil. The approximation is to evaluate $\phi(\alpha)$ at $\alpha_{0}$ and take it outside of the integral

$$
\Delta L(\omega)=\phi\left(\alpha_{0}\right) \Delta L_{0}
$$

Letting $\omega_{0}=\left(2 \alpha_{0}^{2} c+2 \alpha_{0} / \sigma \mu_{0} c\right)$, (8) can be expressed as

$$
\phi(\alpha)=\frac{\frac{j \omega}{\omega_{0}}}{\frac{j \omega}{\omega_{0}}+1+\frac{2 \alpha_{0} \alpha_{1} c}{\left(2 \alpha_{0}^{2} c+2 \alpha_{0}\right)}} .
$$

Noticing that $\alpha_{0} c \ll 1$ is well satisfied for the coil and plate dimension, we have

$$
\begin{aligned}
\omega_{0} & =\frac{2 \alpha_{0}}{\sigma \mu_{0} c} \\
\phi\left(\alpha_{0}\right) & =\frac{\frac{j \omega}{\omega_{0}}}{\frac{j \omega}{\omega_{0}}+1+\alpha_{1} c} .
\end{aligned}
$$

It was found that (12) can be approximated by

$$
\phi\left(\alpha_{0}\right)=\frac{\left(j \frac{\omega}{\omega_{0}}\right)}{\left(1+j \frac{\omega}{\omega_{0}}+0.15 j \sqrt{\frac{\omega}{\omega_{0}}}\right)} .
$$

Note that 0.15 in (13) is an empirical coefficient obtained via trials. It is dependent on the coil geometry, but independent on lift-off and the conductivity of the test plate.
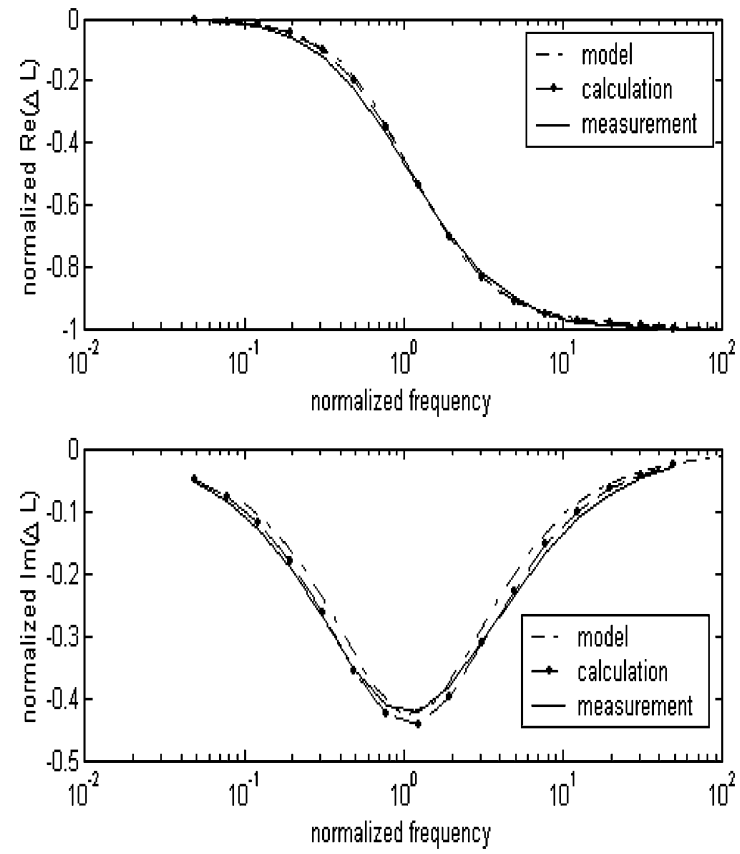

Fig. 5. The comparison of the real and imaginary parts of $\Delta L_{0}$ from the simplified model, the analytic solution and actual measurements.

TABLE I

THE ACTUAL AND INFERRED THICKNESS AND LIFT-OFF

\begin{tabular}{lcccr}
\hline Plate & \multicolumn{2}{c}{ Actual } & \multicolumn{2}{c}{ Inferred } \\
\hline \multirow{2}{*}{ Thickness } & Lift-off & Thickness & Lift-off \\
& $22 \mu \mathrm{m}$ & $1 \mathrm{~mm}$ & $23 \mu \mathrm{m}$ & $1.02 \mathrm{~mm}$ \\
Copper & $66 \mu \mathrm{m}$ & $1.5 \mathrm{~mm}$ & $69 \mu \mathrm{m}$ & $1.54 \mathrm{~mm}$ \\
& $110 \mu \mathrm{m}$ & $2 \mathrm{~mm}$ & $116 \mu \mathrm{m}$ & $2.07 \mathrm{~mm}$ \\
& $18 \mu \mathrm{m}$ & $1 \mathrm{~mm}$ & $21 \mu \mathrm{m}$ & $1.02 \mathrm{~mm}$ \\
Aluminium & $72 \mu \mathrm{m}$ & $1.5 \mathrm{~mm}$ & $71 \mu \mathrm{m}$ & $1.53 \mathrm{~mm}$ \\
& $108 \mu \mathrm{m}$ & $2 \mathrm{~mm}$ & $113 \mu \mathrm{m}$ & $2.04 \mathrm{~mm}$ \\
\hline
\end{tabular}

From (11), it can be concluded that the characteristic angular frequency increases if the conductivity and thickness become smaller, which agrees with observations.

\section{RESULTS}

Fig. 5 shows plots, which provide some verification of the simplified model with analytic solution and measurements. As can be seen, the simple model approximates the frequency-dependent inductance change to a reasonable precision.

The estimation of thickness and lift-off from experimental results takes two steps. (1) Estimate peak $\operatorname{Im} \Delta L_{0}$ and $\omega_{0}$ from experimental measurements. (2) Compare these two values with precalculated peak $\operatorname{Im} \Delta L_{0}$ and $\omega_{0}$ values for a range of thickness and lift-offs; those thickness and lift-off that produce closest peak $\operatorname{Im} \Delta L_{0}$ and $\omega_{0}$ with the experimental measurements are taken as inferred values. Simultaneous thickness and lift-offs inferred from a number of measurements are given in Table I, which verified our model and measurement method. The difference between the actual values and inferred values is 
due to the imperfect modeling of the coil and the error caused by the estimation of peak $\operatorname{Im} \Delta L_{0}$ and $\omega_{0}$ from measurements at a finite number of frequency points. The larger error of the thickness estimation may be due to the large frequency sweeping intervals used. In the process of simplifying the analytical solution, the first approximation $\left(e^{2 \alpha_{1} c}\right.$ to $\left.1+2 \alpha_{1} c\right)$ only holds for small values of $2 \alpha_{1} c$. As thickness increases, the error due to this approximation becomes large. Therefore, with this simplified model, the measurement range for thickness would be between tens and hundreds microns. The measurement range for lift-off is mainly limited by the sensitivity of the coil and measuring instrument, because the inductance change of the coil would be very small for large lift-offs.

Measurement method based on a comprehensive model normally requires calculation and measurement of the inductance change over a large range of frequency. Its principle is to find in a multidimensional table a set of parameters (i.e., thickness and lift-off) for which the calculation is as close as possible to the measured data in a least-squared sense. Therefore, it requires a large storage capability to preserve the calculated data and large computation power to do the search and comparison. The significance in using such a simplified model is that it only requires measurements over a relative small range of frequency (i.e., near the characteristic frequency) and that only one-dimensional (1-D) search is required. These features may facilitate the realization of this method in small portable instruments employing microcontrollers with limited memory and computation power.

\section{CONCLUSION}

Based upon the described simplified model and the measurement method, it is possible to realize a small portable measuring instrument that is capable of providing real-time distance and thickness simultaneously. Future work will involve modeling other coil geometries, configurations (e.g., coils with rectangular cross sections and ferrite-cored coils) and frequency-dependent response for magnetic plates. The field performance of the sensor will be evaluated.

\section{REFERENCES}

[1] D. J. Harrison, L. D. Jones, and S. K. Burke, "Benchmark problems for defect size and shape determination in eddy-current nondestructive evaluation," J. Nondestructive Eval., vol. 15, pp. 21-34, 1996.

[2] D. Placko, H. Clergeot, and E. Santander, "Physical modeling of an eddy current sensor designed for real time distance and thickness measurement in galvanization industry," IEEE Trans. Magn., vol. 25, pp. 2861-2863, 1989.

[3] Y. Nonaka, "A double coil method for simultaneously measuring the resistivity, permeability, and thickness of a moving metal sheet," IEEE Trans. Instrum. Meas., vol. 45, pp. 478-482, 1996.

[4] A. Sethuraman and J. H. Rose, "Rapid inversion of eddy current data for conductivity and thickness of metal coatings," J. Nondestructive Eval., vol. 14, pp. 39-46, 1995.

[5] J. C. Moulder, E. Uzal, and J. H. Rose, "Thickness and conductivity of metallic layers from eddy current measurements," Rev. Sci. Instrum., vol. 63, pp. 3455-3465, 1992.

[6] C. V. Dodd and W. E. Deeds, "Analytical solutions to eddy-current probe-coil problem," J. Appl. Phys., vol. 39, pp. 2829-2839, 1968.

Wuliang Yin received the B.S. and M.S. degrees in electronic measurement and instrumentation from Tianjin University in 1992 and 1995, respectively. He received the Ph.D. degree from Tsinghua University in 1999.

He is currently with Engineering Department, Lancaster University, UK. His current research interests are on electromagnetic sensors and computer tomography.

A. J. Peyton, photograph and biography not available at the time of publication.

Stephen J. Dickinson received the B.Eng. degree in electronic and computer engineering and the $\mathrm{Ph} . \mathrm{D}$. degree in engineering from Lancaster University in 1991 and 1996, respectively.

Following two years working as a Research Associate at Lancaster University, he set up his own limited company developing electronic and software systems mainly developing ultrasonic NDT equipment and software for AEA Sonomatics. He then returned to Lancaster University in 2000 as a Research Associate developing electromagnetic sensors and associated DSP electronics and software, and in 2002, he was appointed as a Lecturer in Electronic Engineering. He has a wide range of knowledge and experience within the areas of embedded computer systems, instrumentation, and control and his current research interests lie within the area of sensors and instrumentation. Currently, he is mainly working on the development electromagnetic sensors and associated algorithms. 